\title{
The assessment of Shin's method for the prediction of creatinine kinase-MB elevation after percutaneous coronary intervention: an intravascular ultrasound study
}

\author{
Eun-Seok Shin • Hector M. Garcia-Garcia • \\ Scot Garg • Jongha Park • Shin-Jae Kim • \\ Patrick W. Serruys
}

Received: 1 October 2010/Accepted: 12 October 2010/Published online: 24 October 2010

(C) Springer Science+Business Media, B.V. 2010

\begin{abstract}
Cardiac enzyme release is common after percutaneous coronary intervention (PCI). At present there is no established relationship between the quantity of necrotic core and dense calcium, as assessed by Shin's method using intravascular ultrasound virtual histology (VH-IVUS), and post-PCI creatinine kinase-MB (CK-MB) elevation. A total of 112 consecutive patients with unstable angina and a normal pre-PCI CK-MB level were imaged using VHIVUS. Patients were divided into 2 groups according to the presence (CK-MB group, $n=22$ ) or absence (non CK-MB group, $n=90)$ of a post-PCI CK-MB elevation $>1.0$ the upper limit of normal $(3.6 \mathrm{ng} / \mathrm{ml})$. Using Shin's method contours were drawn around the IVUS catheter (instead of the lumen), and the vessel. Mean area and volume of necrotic core and dense calcium were significantly greater in CK-MB group than in non CK-MB group $\left(1.7 \pm 0.9 \mathrm{~mm}^{2}\right.$ vs. $0.9 \pm 0.6 \mathrm{~mm}^{2}$, $P<0.001 ; 17.2 \pm 8.8 \mathrm{~mm}^{3}$ vs. $8.8 \pm 5.8 \mathrm{~mm}^{3}, P<$ 0.001 , and $0.9 \pm 0.6 \mathrm{~mm}^{2}$ vs. $0.4 \pm 0.4 \mathrm{~mm}^{2}, P=$ $0.001 ; 9.1 \pm 5.8 \mathrm{~mm}^{3}$ vs. $3.9 \pm 3.7 \mathrm{~mm}^{3}, P<0.001$,
\end{abstract}

E.-S. Shin $(\bowtie) \cdot$ J. Park · S.-J. Kim

Ulsan University Hospital, University of Ulsan College of Medicine, 290-3 Jeonha-dong, Dong-gu, Ulsan 682-714,

South Korea

e-mail: ses@uuh.ulsan.kr

E.-S. Shin · H. M. Garcia-Garcia - S. Garg ·

P. W. Serruys

Thoraxcenter, Erasmus Medical Center, Rotterdam,

The Netherlands respectively). Percent necrotic core and dense calcium areas calculated by external elastic membrane (EEM) area were significantly greater in CK-MB group than in non CK-MB group $(11.9 \pm 5.1$ vs. $6.6 \pm 4.0 \%$, $P<0.001$ and $6.5 \pm 4.0$ vs. $3.0 \pm 2.9 \%, P<0.001$, respectively). In the multivariate analysis, dense calcium volume and percent necrotic core to EEM at the most diseased segment were the only independent predictors of post-PCI CK-MB elevation (odds ratio: 1.22; 95\% confidence interval: $1.09-1.37, P<0.001$ and odds ratio: 1.26; 95\% confidence interval: $1.12-1.43, P<0.001)$. Lesions with a greater amount of dense calcium volume and percent necrotic core to EEM, which can be easily identified and quantified on VH-IVUS analyzed using Shin's method, are responsible for post-PCI CK-MB elevation. With a rapid analyzing time, Shin's method may increase the identification of high risk lesions likely to induce a post-procedural myocardial injury in the catheterization laboratory facilitating on-line clinical decision making.

Keywords Intravascular ultrasound virtual histology · Percutaneous coronary intervention . CK-MB elevation

\section{Introduction}

Myocardial necrosis as indicated by an elevation of creatinine kinase-myoglobin band (CK-MB) occurs 
in 5-30\% of patients following percutaneous coronary intervention (PCI), and is associated with increased mortality even following low rises in CK-MB [1-3]. CK-MB elevation after PCI has been seen linked with side-branch occlusion, thrombus formation, a large atherosclerotic plaque burden as assessed by intravascular ultrasound (IVUS) and an aggressive interventional procedure [4, 5]. A recent study has indicated that a significant relationship exists between plaque components, notably necrotic core, and cardiac enzyme elevation after PCI [6]. Although IVUS Virtual Histology (VH-IVUS) has demonstrated its utility in quantifying coronary plaque components with a high predictive accuracy both in vitro and in vivo [7, 8], it is used infrequently in the catheterization laboratory for the purpose of clinical decision making, which is partly because it is not easy to immediately draw the contours required for analysis. Shin's method for the analysis of VH-IVUS images is described elsewhere; in brief, the method entails drawing the contour of the IVUS catheter instead of the lumen contour, and it is therefore suitable for the measurement of necrotic core and dense calcium. Shin's method is well correlated with the conventional methods employed to measure necrotic core and calcium, and beneficially has a significantly shorter analysis time [9]. The purpose of the present study was to investigate the relationship of pre-PCI necrotic core and calcium content, as determined using VH-IVUS and Shin's method, to CK-MB elevation after PCI.

\section{Methods}

\section{Study population}

This study was conducted in a single Korean center, and enrolled 112 consecutive patients with unstable angina, who had a normal CK-MB level on admission, underwent pre-PCI VH-IVUS and had successful elective coronary artery stenting to a single, native, de novo coronary lesion. All patients had unstable angina, defined as new-onset severe angina, accelerated angina, or angina at rest without ST segment elevation in the ECG. Successful coronary artery stenting was defined as a final residual diameter stenosis $<30 \%$ on visual assessment and normal distal coronary flow at the end of the procedure. Patients with an elevated baseline prePCI CK-MB, a side branch occlusion originating from a stented segment after PCI, or intracoronary thrombus in the target vessel on angiography or IVUS before and after PCI, were excluded. Other exclusions included patients presenting with acute myocardial infarction, patients who had left main disease, chronic total occlusions, patients with impaired left ventricular function on echocardiography, and those who required multi-vessel PCI and/or use of an atherectomy device. All patients received aspirin (100-200 mg) and clopidogrel (300 mg) for at least $24 \mathrm{~h}$ before PCI. All patients provided written informed consent and the study was approved by the hospital's institutional review board.

\section{CK-MB analysis}

Venous blood samples were obtained from all patients at admission, $1 \mathrm{~h}$ before PCI, and at eight hourly intervals for $24 \mathrm{~h}$ post PCI. The upper normal cutoff value for CK-MB according to the assay used in all patients was $3.6 \mathrm{ng} / \mathrm{ml}$. After PCI, patients were divided into 2 groups: post-PCI CKMB $>1$ the upper limit of normal (CK-MB group) versus post-PCI $\mathrm{CKMB} \leq 1$ the upper limit of normal (non CK-MB group).

Angiographic analysis

All studies were reviewed by an experienced angiographer who was blinded as to whether there was a CK-MB elevation. Morphologic characteristics of individual lesions were recorded, and the American College of Cardiology/American Heart Association (ACC/AHA) grading system was used to classify lesions into types $\mathrm{A}, \mathrm{B}$, and $\mathrm{C}$. Lesion length was determined by callipers. Flow in the epicardial coronary arteries was assessed using Thrombolysis in Myocardial Infarction (TIMI) flow grade and the corrected TIMI frame count [10, 11]. Side branch occlusion was assessed thoroughly using side-by-side analysis of cine loops before PCI, and of the final result. Any angiographically visible side branch likely to be directed toward the left ventricular myocardium was considered; only atrial and right ventricular arteries were excluded. Side branch impairment was defined as any non-transient impairment in flow, either TIMI 1 or TIMI 0 [12]. 
VH-IVUS examination and analysis

All pre-PCI VH-IVUS examinations were performed after intracoronary administration of $200 \mu \mathrm{g}$ intracoronary nitroglycerin using a motorized transducer pullback system $(0.5 \mathrm{~mm} / \mathrm{s})$. The 2.9-F IVUS imaging catheter (Eagle Eye, Volcano Corp., Rancho Cordova, California) incorporated a $20-\mathrm{MHz}$ phasedarray transducer. Off-line analyses were performed using VH program software (Volcano Therapeutics, Rancho Cordova, California) by an investigator who was blinded to the patient's clinical characteristics. The VH-IVUS analysis classified and color-coded tissue into 2 components: white (dense calcium), and red (necrotic core) [8]. The region of interest comprised of the most diseased 10-mm segment within the culprit lesion that contained the largest plaque burden. The culprit lesion was defined as the coronary lesion thought responsible for the patient's symptoms and was treated with PCI. The external elastic membrane (EEM) was measured, and percent necrotic core and dense calcium areas or volumes to EEM volume were calculated as necrotic core or dense calcium areas or volumes divided by EEM volume. The VH-IVUS analysis was reported as absolute differences and as a percent necrotic core and dense calcium to EEM.

Shin's method for analysis of necrotic core and dense calcium

Shin's method also used the offline software pcVH 2.2. Instead of measuring the lumen as described above for the conventional method, in Shin's method the lumen contour is drawn around the IVUS catheter, that is, without following the leading edge of the interface lumen intima [9]. When there is a "flare" (ring-down artifact) around the IVUS catheter, this catheter contour must be drawn away from this flare (Fig. 1). The catheter contour was manually detected.

\section{Statistical analysis}

All analyses were performed using SPSS (version 17.0; SPSS Inc, Chicago, Illinois). Discrete variables were presented as percentages and relative frequencies; comparisons were conducted by chi-square statistics or Fisher exact test. Continuous variables were presented as the mean $\pm 1 \mathrm{SD}$; comparisons were conducted by Student $t$ test or nonparametric
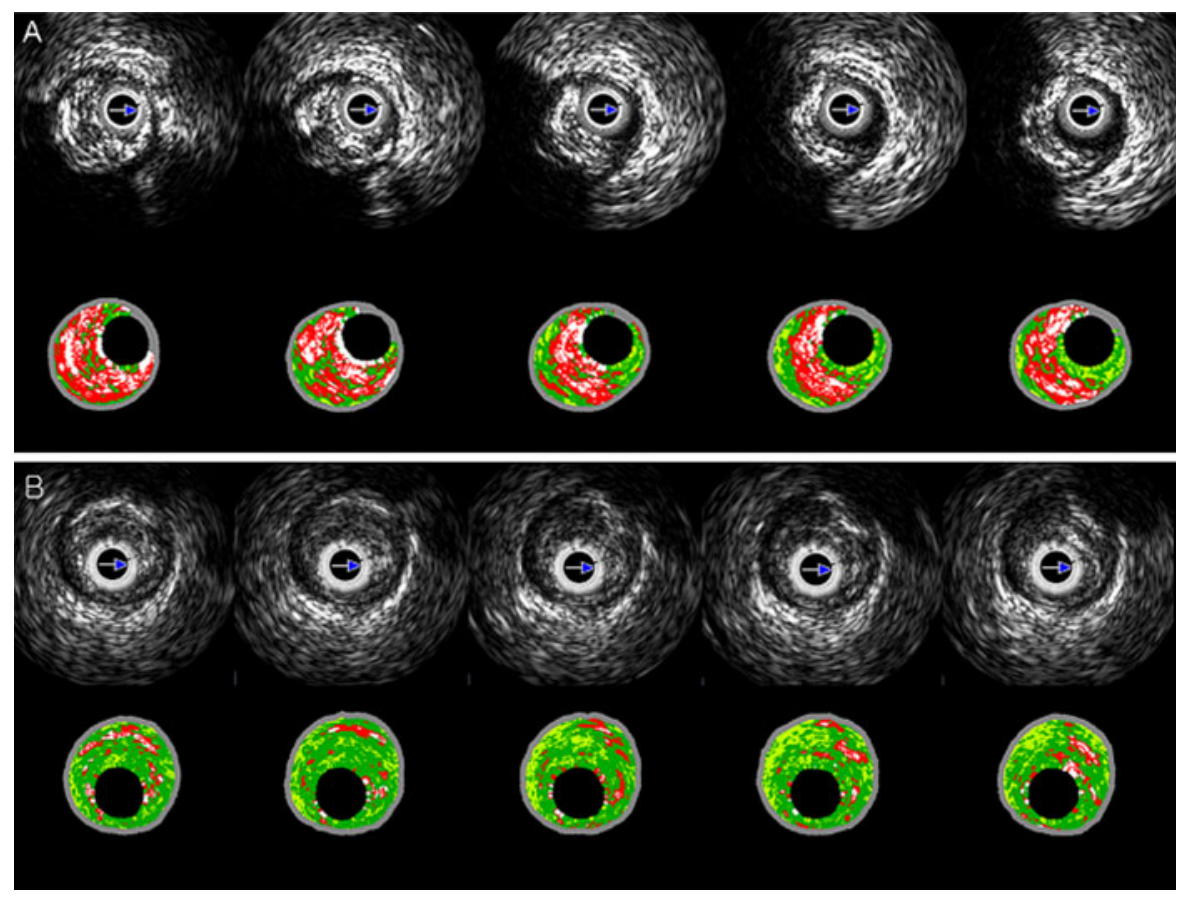

Fig. 1 Comparison of intravascular ultrasound virtual histology images before coronary stenting in patients with CK-MB elevation (A) and non CK-MB elevation (B) 
Wilcoxon test for normally and non-normally distributed data, respectively. A Spearman correlation coefficient was used if the normality assumption was not satisfied. Logistic regression analysis was used to assess demographic, angiographic, procedural and ultrasonographic characteristics associated with CK-MB elevation. Variables with a significance level $<0.2$ in the univariate analysis were considered to be candidate variables for inclusion in the multivariable analysis and a step-down logistic regression was performed. Only variables with a significance level of $<0.05$ were retained in the final models. Odds ratio and the associated $95 \%$ CIs were reported for both the univariable and multivariable logistic regression analyses. Statistical significance was accepted as p less than 0.05 .

\section{Results}

Table 1 shows the baseline characteristics of the patients. Mean age of study subjects was 62 years;
CK-MB group was older than non CK-MB group. Overall, 66 (59\%) were male subjects. There were no differences in levels of lipid or high sensitivity C-reactive protein (hsCRP) between both groups. All patients had taken aspirin and clopidogrel before PCI, whilst Glycoprotein IIb/IIIa inhibitors were not used. The peak CK-MB level was $16.7 \pm 11.7 \mathrm{ng} / \mathrm{ml}$ in CK-MB group and $1.9 \pm 1.1 \mathrm{mg} / \mathrm{ml}$ in non CK-MB group.

Angiographic findings and procedural results

Table 2 shows the angiographic findings and procedural results. The studied vessel was the left anterior descending artery in 66 patients $(58.9 \%)$, the left circumflex artery in $14(12.5 \%)$ and the right coronary artery in 32 (28.6\%). There were more patients with ACC/AHA Type B2 and C lesions in CK-MB group compared with non CK-MB group (86.4 vs. $64.4 \%, P=0.07$ ). All lesions were treated with stent implantation: 75 lesions with sirolimuseluting stents, 2 lesion with paclitaxel-eluting stent,
Table 1 Baseline characteristics
Values are presented as number $(\%)$ or mean $\pm \mathrm{SD}$. $M I$ myocardial infarction, $P C I$ percutaneous coronary intervention, $C A B G$ coronary artery bypass grafting, $H D L$ high-density lipoprotein, $L D L$ cholesterol low-density lipoprotein, $h s C R P$ high sensitivity C-reactive protein

\begin{tabular}{|c|c|c|c|}
\hline & CK-MB $(n=22)$ & Non CK-MB $(n=90)$ & $P$ \\
\hline Age, years & $65.5 \pm 8.3$ & $61.4 \pm 8.3$ & 0.06 \\
\hline Male & $11(50)$ & $55(61.1)$ & 0.35 \\
\hline \multicolumn{4}{|l|}{ History } \\
\hline Previous MI & $2(9.1)$ & $4(4.4)$ & 0.34 \\
\hline Previous PCI & $3(13.6)$ & $17(18.9)$ & 0.76 \\
\hline Previous CABG & $2(9.1)$ & $1(1.1)$ & 0.1 \\
\hline \multicolumn{4}{|l|}{ Risk factors } \\
\hline Hypertension & $16(72.7)$ & $46(51.1)$ & 0.09 \\
\hline Hypercholesterolemia & $14(63.6)$ & $48(53.3)$ & 0.48 \\
\hline Diabetes mellitus & $5(22.7)$ & $29(32.2)$ & 0.45 \\
\hline Current smoking & $1(4.5)$ & $23(25.6)$ & 0.04 \\
\hline Previous statin use & $6(27.3)$ & $19(21.1)$ & 0.57 \\
\hline Before PCI aspirin use & $22(100)$ & $90(100)$ & $>0.99$ \\
\hline Before PCI clopidogrel use & $22(100)$ & $90(100)$ & $>0.99$ \\
\hline Glycoprotein IIb/IIIa inhibitor use & 0 & 0 & \\
\hline Ejection fraction, \% & $63.5 \pm 5.3$ & $63.4 \pm 7.1$ & 0.92 \\
\hline Total cholesterol, mg/dl & $192.9 \pm 39.3$ & $189.0 \pm 43.3$ & 0.70 \\
\hline Triglyceride, mg/dl & $132.9 \pm 55.1$ & $151.5 \pm 82.5$ & 0.32 \\
\hline HDL cholesterol, mg/dl & $40.6 \pm 6.1$ & $42.1 \pm 13.9$ & 0.63 \\
\hline LDL cholesterol, mg/dl & $116.6 \pm 33.4$ & $106.3 \pm 36.7$ & 0.63 \\
\hline Serum creatinine, $\mathrm{mg} / \mathrm{dl}$ & $1.04 \pm 0.12$ & $1.11 \pm 0.55$ & 0.53 \\
\hline hsCRP, mg/L & $2.0 \pm 3.1$ & $1.8 \pm 3.6$ & 0.83 \\
\hline Peak CKMB, ng/ml & $16.7 \pm 11.7$ & $1.9 \pm 1.1$ & $<0.001$ \\
\hline
\end{tabular}


Table 2 Angiographic findings and procedural results
Values are presented as number $(\%)$ or mean $\pm \mathrm{SD}$. $L A D$ left anterior descending artery, $L C X$ left circumflex artery, $R C A$ right coronary artery

\begin{tabular}{|c|c|c|c|}
\hline & CK-MB $(n=22)$ & Non CK-MB $(n=90)$ & $P$ \\
\hline \multicolumn{4}{|l|}{ Study vessel } \\
\hline LAD & $11(50)$ & $55(61.1)$ & 0.35 \\
\hline LCX & $5(22.7)$ & $9(10)$ & 0.15 \\
\hline RCA & $6(27.3)$ & $26(28.9)$ & $>0.99$ \\
\hline Lesion type, B2 and C & $19(86.4)$ & $58(64.4)$ & 0.07 \\
\hline Side branch & $14(63.6)$ & $54(60.0)$ & 0.81 \\
\hline Lesion length, mm & $26.5 \pm 19.9$ & $24.2 \pm 17.5$ & 0.59 \\
\hline Degree of stenosis & $63.1 \pm 19.9$ & $59.8 \pm 17.9$ & 0.46 \\
\hline Stent type & & & 0.65 \\
\hline Sirolimus-eluting stent & $13(59.1)$ & $62(68.9)$ & \\
\hline Paclitaxel-eluting stent & 0 & $2(2.2)$ & \\
\hline Everolimus-eluting stent & $5(22.7)$ & $13(14.4)$ & \\
\hline Bare-metal stent & $4(18.2)$ & $13(14.4)$ & \\
\hline Number of stents & $1.6 \pm 0.6$ & $1.3 \pm 0.6$ & 0.11 \\
\hline Stent diameter, $\mathrm{mm}$ & $3.3 \pm 0.3$ & $3.4 \pm 0.3$ & 0.23 \\
\hline Stent length, mm & $32.8 \pm 16.3$ & $30.9 \pm 18.8$ & 0.62 \\
\hline Stent-balloon maximum pressure, atm & $14.1 \pm 3.1$ & $15.1 \pm 3.4$ & 0.19 \\
\hline Post-stent balloon use & $19(86.4)$ & $72(80)$ & 0.76 \\
\hline Post-stent balloon size, mm & $3.6 \pm 0.4$ & $3.9 \pm 0.5$ & 0.46 \\
\hline Post-stent balloon maximum pressure, atm & $17.2 \pm 1.9$ & $16.9 \pm 3.0$ & 0.64 \\
\hline Post-PCI TIMI flow grade $0 / 1$ & 0 & 0 & \\
\hline Post-PCI corrected TIMI frame count & $16.0 \pm 7.3$ & $12.6 \pm 5.1$ & 0.07 \\
\hline
\end{tabular}

18 lesions with everolimus-eluting stents and 17 lesions with bare-metal stents. There was no significant difference between groups in the frequency of side branches, the lesion length and degree of stenosis. The size of balloon used for post-stenting dilatation, and the maximum pressure used was similar for both groups. No patients had a post-PCI TIMI flow grade $0 / 1$, and post-PCI corrected TIMI frame count was not significantly different between groups $(16.0 \pm 7.3$ vs. $12.6 \pm 5.1, P=0.07)$.

\section{IVUS results}

Table 3 shows the geometrical and compositional findings. Patients in both groups had a comparable plaque burden $(67.1 \pm 7.8 \%$ in CK-MB group and $66.2 \pm 8.3 \%$ in non CK-MB group, $P=0.63$ ), mean EEM area and remodeling index. The mean necrotic core and dense calcium area were significantly greater in CK-MB group (Fig. 2). In addition, the necrotic core and dense calcium volumes were significantly greater in the CK-MB group than in the non CK-MB group. Percent necrotic core area and
Table 3 Geometrical and compositional characteristics

\begin{tabular}{|c|c|c|c|}
\hline & $\begin{array}{l}\text { CK-MB } \\
(n=22)\end{array}$ & $\begin{array}{l}\text { Non CK-MB } \\
(n=90)\end{array}$ & $P$ \\
\hline Remodelling index & $1.1 \pm 0.3$ & $1.0 \pm 0.2$ & 0.16 \\
\hline \multicolumn{4}{|c|}{ Volumetric analysis, $\mathrm{mm}^{3}$} \\
\hline EEM & $143.2 \pm 37.5$ & $137.2 \pm 33.1$ & 0.46 \\
\hline Dense calcium & $9.1 \pm 5.8$ & $3.9 \pm 3.7$ & $<0.001$ \\
\hline Necrotic core & $17.2 \pm 8.8$ & $8.8 \pm 5.8$ & $<0.001$ \\
\hline \multicolumn{4}{|l|}{ Mean area, $\mathrm{mm}^{2}$} \\
\hline EEM & $14.1 \pm 3.7$ & $13.6 \pm 3.2$ & 0.48 \\
\hline Dense calcium & $0.9 \pm 0.6$ & $0.4 \pm 0.4$ & 0.001 \\
\hline Necrotic core & $1.7 \pm 0.9$ & $0.9 \pm 0.6$ & $<0.001$ \\
\hline \multicolumn{4}{|c|}{ Percent area to EEM, \% } \\
\hline Dense calcium & $6.5 \pm 4.0$ & $3.0 \pm 2.9$ & $<0.001$ \\
\hline Necrotic core & $11.9 \pm 5.1$ & $6.6 \pm 4.0$ & $<0.001$ \\
\hline
\end{tabular}

Values are presented mean \pm SD. EEM external elastic membrane

volume, and percent dense calcium area and volume to EEM were also significantly greater in the CK-MB group compared to the non CK-MB group. A representative case of VH-IVUS is shown in Fig. 1. 

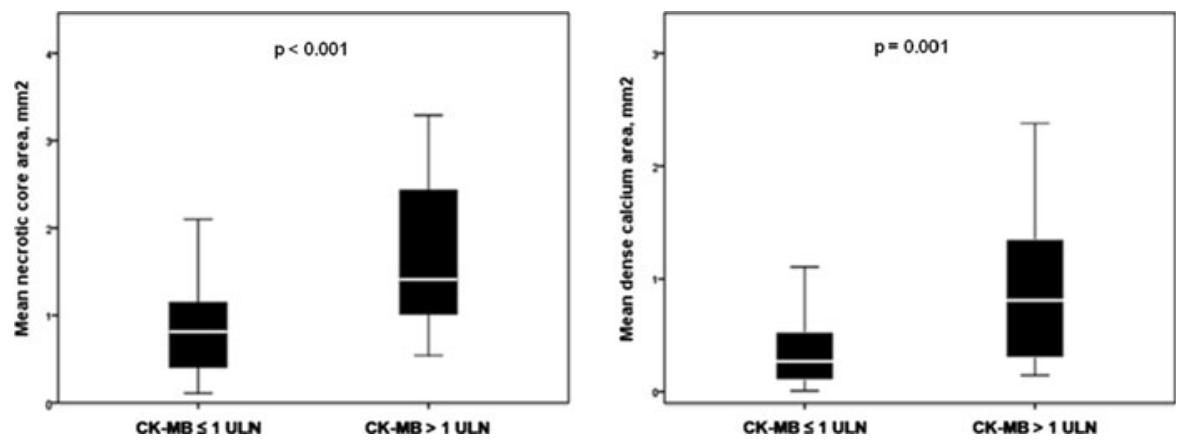

Fig. 2 Comparisons of mean necrotic core and dense calcium areas before coronary stenting with CK-MB elevation and non CK-MB elevation

\section{Correlations to peak CK-MB level}

The peak CK-MB level correlated with: (1) necrotic core volume $(r=0.438, P<0.001)$ and mean necrotic core area $(r=0.428, P<0.001)$; (2) dense calcium volume $(r=0.414, P<0.001)$ and mean dense calcium area $(r=0.428, P<0.001)$ (3) percent necrotic core area to EEM $(r=0.384, P<0.001)$; (4) percent dense calcium area to $\operatorname{EEM}(r=0.358$, $P<0.001$ ) at the most diseased segment (Fig. 3).

Independent predictors of post-PCI CK-MB elevation

Demographic, angiographic, procedural and ultrasonographic variables as they relate to CK-MB elevation are shown in Table 4. Those variables that were independently predictive of CK-MB elevation are shown in Table 5. Dense calcium volume and percent necrotic core to EEM were the only independent predictors of post-PCI CK-MB elevation (odds ratio: 1.22; $95 \%$ confidence interval: $1.09-1.37, P=0.001$ and odd ratio: 1.26; $95 \%$ confidence interval: $1.12-1.43, P<0.001)$. The area under the receiveroperator characteristic curve for percent necrotic core area to EEM at the most diseased segment predicting post-PCI CK-MB elevation $>1$ the upper limit of normal was 0.791 (Fig. 4).

\section{Discussion}

The present study is the first report to demonstrate that necrotic core and dense calcium measured by Shin's method using pre-PCI VH-IVUS is associated with post-PCI CK-MB elevation. CK-MB elevation post PCI has been validated as a marker of short- and long-term prognosis [2, 13-15]. The most common definition of peri-procedural MI is a CK-MB elevation $\geq 3$ times the upper limit of normal, however, this is only an arbitrary cutoff [3]. A recent study showed that the lack of substantial agreement between cardiac enzymes and late gadolinium enhancement by cardiac MRI for the diagnosis of small-sized periprocedural myocardial damage after PCI [16]. However, contrast enhanced MRI has demonstrated that even low levels of CK-MB elevation can correspond to discrete areas of micro-infarction [17]. Alternatively, even small, asymptomatic elevations of CK-MB have been clearly associated with a poorer long-term outcome [1, 18, 19]. The aim of the current study was to demonstrate the association between CK-MB elevation and plaque composition, and therefore those patients with other factors known to increase CK-MB levels such as side-branch occlusion, intracoronary thrombus and no-reflow after stent implantation were excluded. Recently, several studies have shown the relationship between VH-IVUS findings and post-PCI distal embolization. Kawaguchi et al. studied high-risk plaque and distal embolization after stent deployment in patients with acute ST-segment elevation myocardial infarction with VH-IVUS. Results showed that the volume of necrotic core clearly predicted ST-segment re-elevation, which was used as a surrogate to evaluate distal embolization in this study after stent deployment [20]. Kawamoto et al. studied the relationship between coronary plaque components and the small embolic particles produced during stenting, and also examined the influence of this on the coronary microcirculation using VH-IVUS. They 
Fig. 3 Correlations of CKMB level and A necrotic core volume, $\mathbf{B}$ dense calcium volume, $\mathbf{C}$ mean necrotic core area, $\mathbf{D}$ mean dense calcium area,

E percent necrotic core area to EEM, $\mathbf{F}$ percent dense calcium area to EEM (EEM external elastic membrane)
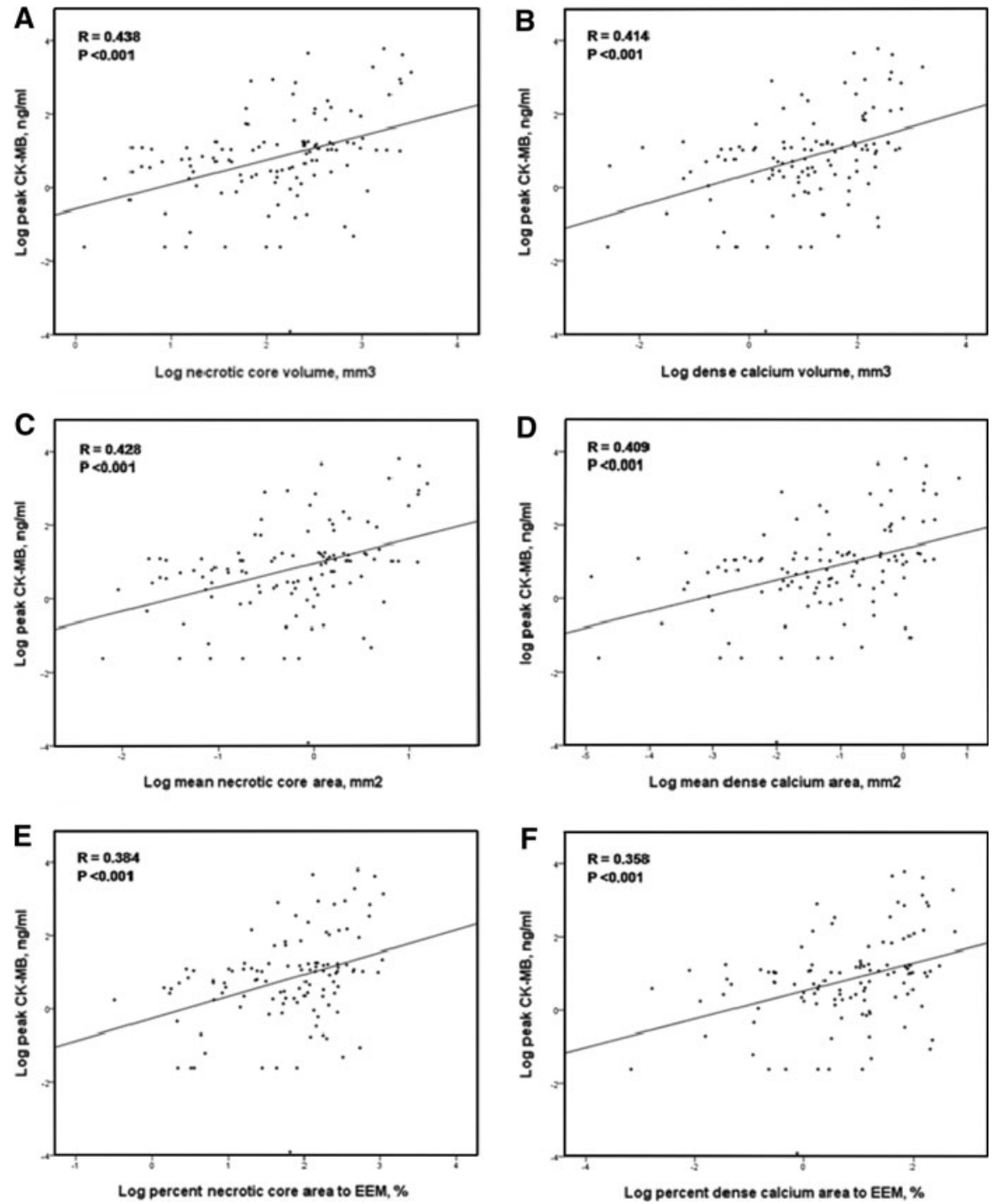

showed that the necrotic core component was related to liberation of small embolic particles during coronary stenting, which resulted in the poorer recovery of coronary flow reserve velocity [21]. Although VH-IVUS may play an important role in detecting which lesions are at high risk for myocardial necrosis after PCI, it is not used routinely in the catheterization laboratory for clinical decision making, which is partly because it is not easy to immediately draw the contours. In particular, lumen contour detection takes a long time and it is especially challenging in patients with high risk lesions (i.e., acute coronary syndrome) because there is no clear separation between luminal thrombi and intima [9]. Shin's method is well correlated with conventional methods for the measurement of necrotic core and calcium content, whilst also being significantly quicker to perform [9]. In the present study, post-PCI CK-MB elevation occurred in lesions with a large dense calcium volume and percent necrotic core area to EEM at the most diseased $10 \mathrm{~mm}$ segment of the lesion. The CK-MB elevation correlated with necrotic core volume and area, dense calcium volume and area, percent necrotic core area and percent dense calcium area to EEM at the most diseased segment. In the multivariate analysis, dense calcium volume and percent necrotic core area to EEM at the most diseased segment were the only independent 
Table 4 Univariate analysis of CK-MB elevation (CK$\mathrm{MB}>3.6 \mathrm{ng} / \mathrm{ml})$
EEM external elastic membrane

\begin{tabular}{|c|c|c|c|}
\hline & OR & $95 \% \mathrm{CI}$ & $P$ \\
\hline \multicolumn{4}{|l|}{ Demographic } \\
\hline Age & 1.05 & $0.10-1.10$ & 0.08 \\
\hline Male & 0.64 & $0.25-1.62$ & 0.34 \\
\hline Previous MI & 2.15 & $0.37-12.57$ & 0.40 \\
\hline Previous PCI & 0.68 & $0.18-2.56$ & 0.57 \\
\hline Previous $\mathrm{CABG}$ & 8.90 & $0.77-103.03$ & 0.08 \\
\hline Hypertension & 2.55 & $0.92-7.11$ & 0.07 \\
\hline Hypercholesterolemia & 1.50 & $0.57-3.92$ & 0.41 \\
\hline Diabetes mellitus & 0.62 & $0.21-1.84$ & 0.39 \\
\hline Current smoking & 0.14 & $0.02-1.09$ & 0.06 \\
\hline \multicolumn{4}{|l|}{ Angiographic } \\
\hline Lesion type, B2 and C & 3.49 & $0.96-12.72$ & 0.06 \\
\hline Side branch & 1.17 & $0.44-3.06$ & 0.75 \\
\hline Lesion length & 1.01 & $0.98-1.03$ & 0.59 \\
\hline Degree of stenosis & 1.01 & $0.98-1.04$ & 0.46 \\
\hline \multicolumn{4}{|l|}{ Procedural } \\
\hline Number of stents & 1.79 & $0.86-3.71$ & 0.12 \\
\hline Stent-balloon maximum pressure & 0.91 & $0.80-1.05$ & 0.20 \\
\hline Post-stent balloon use & 1.58 & $0.42-5.94$ & 0.50 \\
\hline Post-stent balloon maximum pressure & 1.04 & $0.86-1.25$ & 0.71 \\
\hline \multicolumn{4}{|l|}{ Intravascular ultrasound } \\
\hline Remodelling index & 5.26 & $0.52-53.17$ & 0.16 \\
\hline EEM volume & 1.01 & $0.99-1.02$ & 0.45 \\
\hline Dense calcium volume & 1.26 & $1.12-1.40$ & $<0.001$ \\
\hline Necrotic core volume & 1.16 & $1.08-1.25$ & $<0.001$ \\
\hline Mean EEM area & 1.05 & $0.91-1.21$ & 0.47 \\
\hline Mean dense calcium area & 10.22 & $3.29-31.74$ & $<0.001$ \\
\hline Mean necrotic core area & 4.65 & $2.23-9.67$ & $<0.001$ \\
\hline Percent dense calcium to EEM & 1.32 & $1.14-1.54$ & $<0.001$ \\
\hline Percent necrotic core to EEM & 1.29 & $1.14-1.45$ & $<0.001$ \\
\hline
\end{tabular}

Table 5 Multivariable predictors of CK-MB elevation (CK$\mathrm{MB}>3.6 \mathrm{ng} / \mathrm{ml}$ )

\begin{tabular}{lllr}
\hline & OR & $95 \%$ CI & \multicolumn{1}{l}{$P$} \\
\hline Dense calcium volume & 1.22 & $1.09-1.37$ & 0.001 \\
Percent necrotic core to EEM & 1.26 & $1.12-1.43$ & $<0.001$ \\
\hline
\end{tabular}

EEM external elastic membrane

predictors of post-PCI CK-MB elevation (odds ratio: 1.22; $95 \%$ confidence interval: $1.09-1.37, P=0.001$ and odd ratio: 1.26 ; $95 \%$ confidence interval: 1.12-1.43, $P<0.001)$. Thus, Shin's method may be a useful modality for detecting lesions at risk for myocardial necrosis during PCI.

\section{Clinical implications}

Post-PCI myonecrosis has been shown to be significantly reduced following the administration before PCI of pharmacological treatments such as glycoprotein IIb/IIIa inhibitors [22-24] and statin therapy [25], or the use of distal emboli protection devices [26]. However, the precise indications for using these interventions have not been clearly established. 


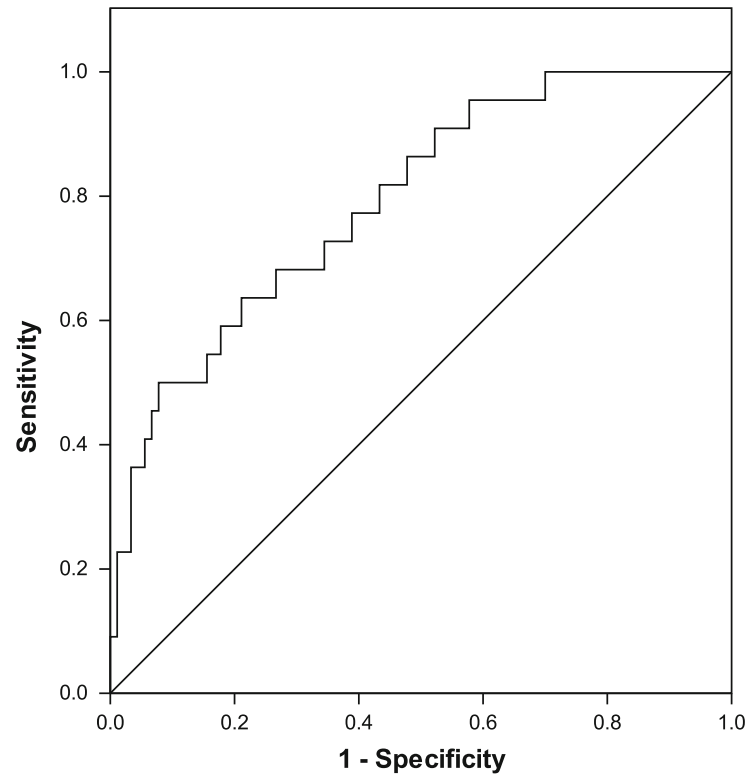

Fig. 4 Receiver-operator characteristic curve for percent necrotic core area to EEM predicting CK-MB level; the area under the receiver-operator characteristic curve for percent necrotic core area to EEM at the most diseased segment predicting post-PCI CK-MB elevation $>1$ the upper limit of normal was 0.791 (EEM external elastic membrane)

Measurement of necrotic core by Shin's method may increase the identification of those lesions likely to induce a post-procedural myocardial injury, and therefore indicate which lesions would benefit most from these interventions for myocardial protection.

\section{Limitations}

First, this study is based on a relatively small sample size and arbitrary definition of post-PCI myocardial injury. Second, diffuse atherosclerotic disease in the target lesion are important factor affecting postprocedural CK-MB elevation [5]. We did not evaluate total plaque volume in the target lesion and its relationship with post-PCI CK-MB elevation. Third, this was a retrospective single-center study. The results of this study need to be verified by multicenter, prospective studies. Lastly, Shin's method does not offer information about the location of the necrotic core and dense calcium in relation to the lumen.

\section{Conclusions}

Lesions with a higher content of dense calcium and percent necrotic core to EEM, which can be easily identified and quantified on VH-IVUS analyzed using Shin's method, are responsible for post-PCI CK-MB elevation. With a shorter analysis time, Shin's method may increase the identification of high risk lesions likely to induce a post-procedural myocardial injury in the catheterization laboratory facilitating a real-time clinical decision.

Conflict of interest We declare that there is no conflict of interest for any author.

\section{References}

1. Topol EJ, Ferguson JJ, Weisman HF et al (1997) Longterm protection from myocardial ischemic events in a randomized trial of brief integrin \{beta\} 3 blockade with percutaneous coronary intervention. JAMA 278:479-484

2. Tardiff BE, Califf RM, Tcheng JE et al (1999) Clinical outcomes after detection of elevated cardiac enzymes in patients undergoing percutaneous intervention. J Am Coll Cardiol 33:88-96

3. Califf RM, Abdelmeguid AE, Kuntz RE et al (1998) Myonecrosis after revascularization procedures. J Am Coll Cardiol 31:241-251

4. Ricciardi MJ, Davidson CJ, Gubernikoff G et al (2003) Troponin I elevation and cardiac events after percutaneous coronary intervention. Am Heart J 145:522-528

5. Mehran R, Dangas G, Mintz GS et al (2000) Atherosclerotic plaque burden and CK-MB enzyme elevation after coronary interventions: intravascular ultrasound study of 2256 patients. Circulation 101:604-610

6. Hong YJ, Mintz GS, Kim SW et al (2009) Impact of plaque composition on cardiac troponin elevation after percutaneous coronary intervention: an ultrasound analysis. JACC Cardiovasc Imaging 2:458-468

7. Nasu K, Tsuchikane E, Katoh O et al (2006) Accuracy of in vivo coronary plaque morphology assessment: a validation study of in vivo virtual histology compared with in vitro histopathology. J Am Coll Cardiol 47:2405-2412

8. Nair A, Kuban BD, Tuzcu EM, Schoenhagen P, Nissen SE, Vince DG (2002) Coronary plaque classification with intravascular ultrasound radiofrequency data analysis. Circulation 106:2200-2206

9. Shin ES, Garcia-Garcia HM, Serruys PW (2010) A new method to measure necrotic core and calcium content in coronary plaques using intravascular ultrasound radiofrequency-based analysis. Int $\mathrm{J}$ Cardiovasc Imaging 26: 387-396

10. The Thrombolysis in Myocardial Infarction (TIMI) trial (1985) Phase I findings. TIMI study group. N Engl J Med 312:932-936 
11. Gibson CM, Cannon CP, Daley WL et al (1996) TIMI frame count: a quantitative method of assessing coronary artery flow. Circulation 93:879-888

12. Porto I, Selvanayagam JB, Van Gaal WJ et al (2006) Plaque volume and occurrence and location of periprocedural myocardial necrosis after percutaneous coronary intervention: insights from delayed-enhancement magnetic resonance imaging, thrombolysis in myocardial infarction myocardial perfusion grade analysis, and intravascular ultrasound. Circulation 114:662-669

13. Hong MK, Mehran R, Dangas G et al (1999) Creatine kinase-MB enzyme elevation following successful saphenous vein graft intervention is associated with late mortality. Circulation 100:2400-2405

14. Akkerhuis KM, Alexander JH, Tardiff BE et al (2002) Minor myocardial damage and prognosis: are spontaneous and percutaneous coronary intervention-related events different? Circulation 105:554-556

15. Kong TQ Jr, Davidson CJ, Meyers SN, Tauke JT, Parker MA, Bonow RO (1997) Prognostic implication of creatine kinase elevation following elective coronary artery interventions. JAMA 277:461-466

16. Locca D, Bucciarelli-Ducci C, Ferrante G et al (2010) New universal definition of myocardial infarction applicable after complex percutaneous coronary interventions? JACC Cardiovasc Interv 3:950-958

17. Ricciardi MJ, Wu E, Davidson CJ et al (2001) Visualization of discrete microinfarction after percutaneous coronary intervention associated with mild creatine kinase-MB elevation. Circulation 103:2780-2783

18. Brener SJ, Lytle BW, Schneider JP, Ellis SG, Topol EJ (2002) Association between CK-MB elevation after percutaneous or surgical revascularization and three-year mortality. J Am Coll Cardiol 40:1961-1967
19. Bhatt DL, Topol EJ (2005) Periprocedural cardiac enzyme elevation predicts adverse outcomes. Circulation 112: 906-922

20. Kawaguchi R, Oshima S, Jingu M et al (2007) Usefulness of virtual histology intravascular ultrasound to predict distal embolization for ST-segment elevation myocardial infarction. J Am Coll Cardiol 50:1641-1646

21. Kawamoto T, Okura H, Koyama Y et al (2007) The relationship between coronary plaque characteristics and small embolic particles during coronary stent implantation. J Am Coll Cardiol 50:1635-1640

22. (2000) Novel dosing regimen of eptifibatide in planned coronary stent implantation (ESPRIT): a randomised, placebo-controlled trial. Lancet 356:2037-2044

23. Bonz AW, Lengenfelder B, Strotmann J et al (2002) Effect of additional temporary glycoprotein IIb/IIIa receptor inhibition on troponin release in elective percutaneous coronary interventions after pretreatment with aspirin and clopidogrel (TOPSTAR trial). J Am Coll Cardiol 40: 662-668

24. Valgimigli M, Percoco G, Barbieri D et al (2004) The additive value of tirofiban administered with the high-dose bolus in the prevention of ischemic complications during high-risk coronary angioplasty: The advance trial. J Am Coll Cardiol 44:14-19

25. Briguori C, Visconti G, Focaccio A et al (2009) Novel approaches for preventing or limiting events (Naples) II trial: impact of a single high loading dose of atorvastatin on periprocedural myocardial infarction. J Am Coll Cardiol 54:2157-2163

26. Angelini A, Rubartelli P, Mistrorigo F et al (2004) Distal protection with a filter device during coronary stenting in patients with stable and unstable angina. Circulation 110: $515-521$ 